\title{
Diffuse Leiomyomatosis: Complete Myomectomy for Innumerable Small Nodules to Achieve Fertility Sparing and Childbearing
}

\author{
Ikuo Konishi, MD, $\mathrm{PhD}^{1}$ \\ ${ }^{1}$ National Hospital Organization, Kyoto Medical Center, Kyoto, Japan \\ Surg J 2020;6(suppl S1):S50-S57.
}

\begin{abstract}
Address for correspondence Ikuo Konishi, MD, PhD, National Hospital Organization, Kyoto Medical Center, 1-1 Fukakusa Mukaihata-cho, Fushimiku, Kyoto 612-8555, Japan (e-mail: konishi@kuhp.kyoto-u.ac.jp).
\end{abstract}

\author{
Abstract \\ Keywords \\ - diffuse \\ leiomyomatosis \\ - extensive \\ myomectomy \\ - longitudinal incision \\ of uterus \\ - GnRH agonist \\ - MRI \\ - junctional zone
}

\begin{abstract}
Diffuse leiomyomatosis is a rare condition among benign smooth muscle neoplasms of the uterus, being defined as innumerable small myomatous nodules that mainly occur in the submucosal area. Young women with this disease suffer from menorrhagia, pains, and infertility. It is essential to perform extensive myomectomy after opening the endometrial cavity by a deep, median, longitudinal incision of the uterine corpus, followed by careful suture and reconstruction of the uterus for fertility spearing and childbearing in young women with this disease. So far, there have been no experiences of the uterine rupture during pregnancy even after such myomectomy, but Caesarean section is recommended for safe delivery.
\end{abstract}

\section{Diffuse Leiomyomatosis}

Diffuse leiomyomatosis is an unusual condition in which innumerable small smooth muscle nodules produce symmetrical enlargement of the uterus. The uterus may be greatly enlarged, weighting up to $1,000 \mathrm{~g}$. Each nodule ranges from microscopic to $3 \mathrm{~cm}$ in size, but mostly less than $1 \mathrm{~cm}$ in diameter. They are composed of uniform, bland, spindled-shaped smooth muscle cells, and are less circumscribed than typical leiomyoma. Usually, small nodules occur mainly in the submucosal area, and, therefore, they are recognized as extensive submucosal myomas by ultrasonography or magnetic resonance imaging (MRI). Diffuse leiomyomatosis occur in young women at the age of 30's and produce severe symptoms, such as hypermenorrhea and dysmenorrhea. All of them suffer from iron-deficiency anemia and also use analgesics during menstruation. They frequently have a past history undergoing myomectomy. However, traditional surgical approaches by open laparotomy, laparoscopy, and hysteroscopy usually result in an incomplete myomectomy, and the patient will complain again of the same symptoms soon after the surgery due to recurrence. Finally, the patients tend to undergo hysterectomy without childbearing. Thus, diffuse leiomyomatosis is an important clinical entity and a difficult-to-cure disease in young women who desires fertility spearing. Gynecologists are asked enthusiastically by the patient to remove numerous submucosal myomas as complete as possible. In this text, a novel and radical surgical approach for diffuse leiomyomatosis is described. ${ }^{1}$

\section{Preoperative Evaluation}

MRI is absolutely needed to diagnose diffuse leiomyomatosis. T2-weighted image will disclose numerous small nodules mainly in the junctional zone of the uterine corpus (Fig. 1A, $B)$. Therefore, this disease is recognized by gynecologists as extensive submucosal myomas of the uterus. Several nodules may be present in the subserosal area, but this is not frequent.

\section{Hormonal Treatment before Surgery}

It is advised to prescribe gonadotropin-releasing hormone (GnRH) agonist or antagonist for 3 to 6 months before the
DOI https://doi.org/ 10.1055/s-0039-1693709. ISSN 2378-5128.
Copyright $\odot 2020$ by Thieme Medical Publishers, Inc., 333 Seventh Avenue, New York, NY 10001, USA. Tel: +1(212) 760-0888.
License terms

()(1) $\circledast$ 
surgery. During such hormonal treatment, the uterus and the nodules become smaller, and the surgery will become much easier. The second MRI after the hormonal treatment is essential for preoperative planning of the surgery (-Fig. 1C, D).

\section{Tips and Warnings-What is Junctional Zone?}

In T2-weighted MRI, the uterine corpus shows the three distinct zonal layers, that is, the endometrium which is characterized by high-intensity zone along the cavity, the outer myometrial zone showing relatively high intensity under the serosa, and the junctional zone with low intensity between the endometrium and the outer
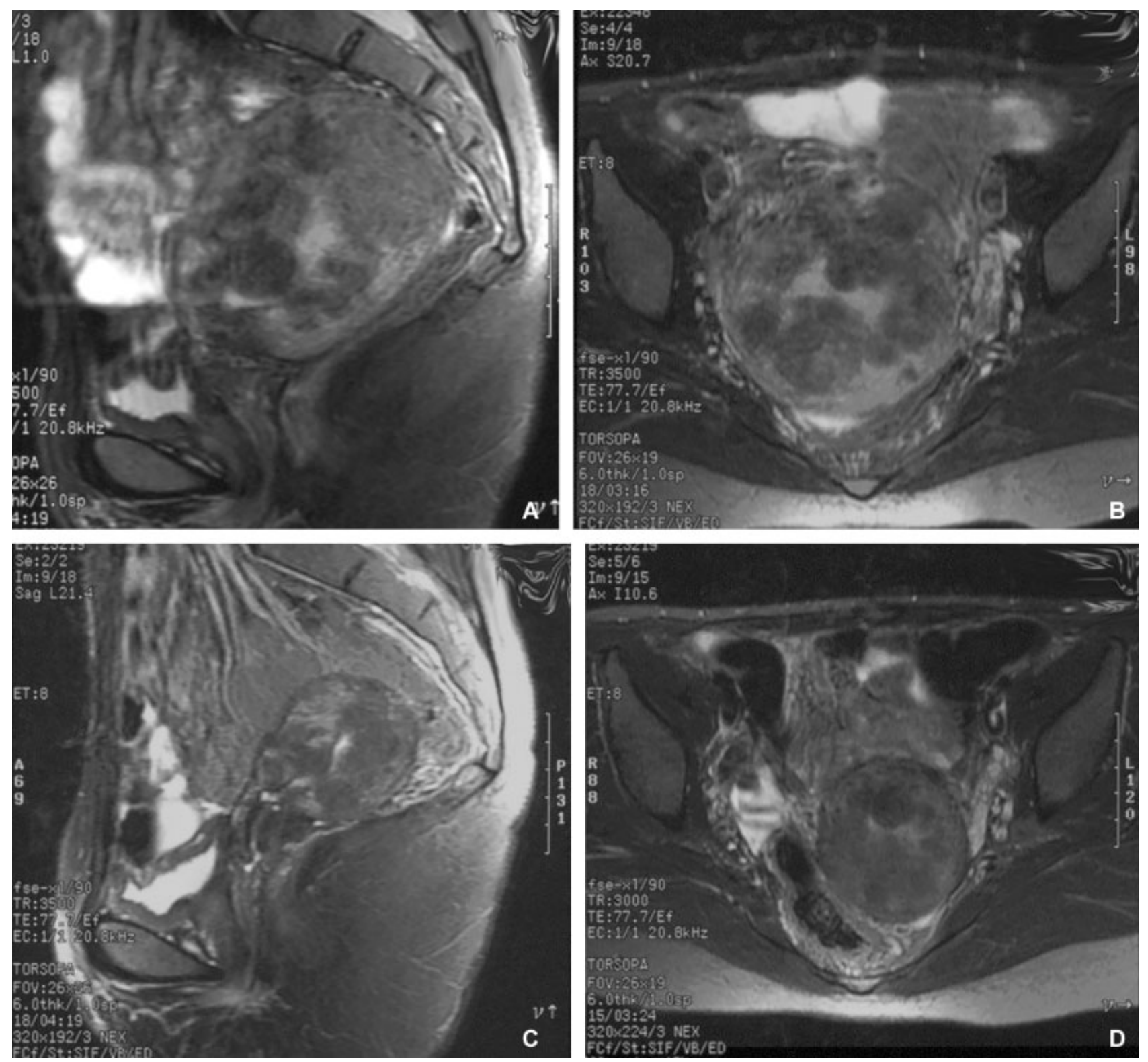

Fig. 1 MRI T2-weighted findings of diffuse leiomyomatosis: nnumerable small nodules are present in the junctional zone, and protruded into the uterine cavity (A, B). After treatment with four cycles of $\mathrm{GnRH}$ agonist, the uterus and myomatous nodules decrease in size(C, D).(A, C) Sagittal section, (B, D) transverse section. 


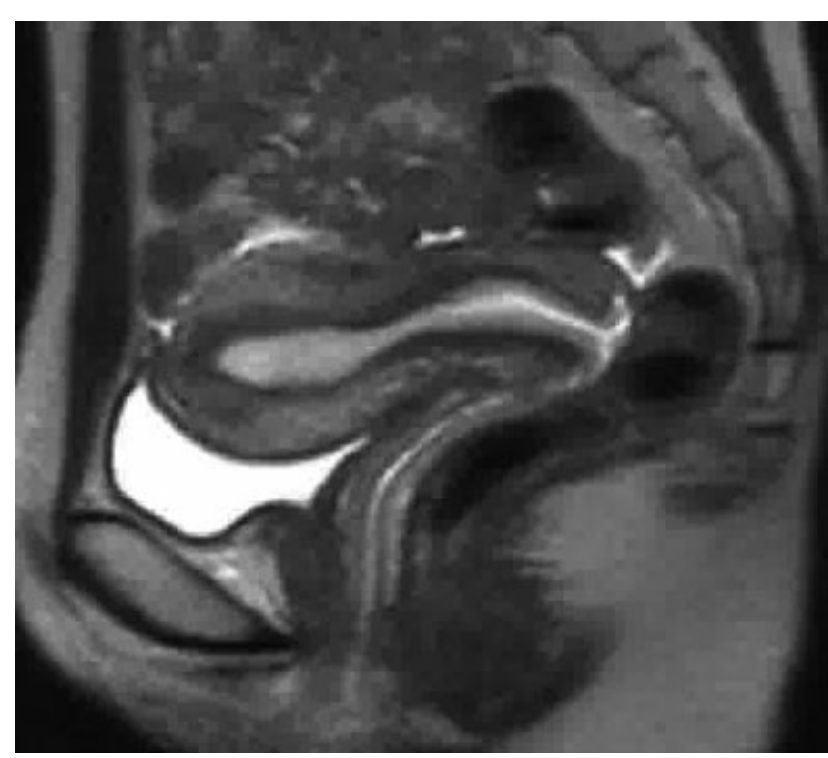

Fig. 2 Junctional zone of the uterus: in T2-weighted MRI figures, the uterine corpus is composed by three different zones. The endometrium appears as the innermost, high-intensity zone. The outer subserosal zone of the myometrium shows an intermediately high intensity. The low-intensity zone between the endometrium and the outer myometrium is called as "junctional zone", but this is actually the inner part of the myometrium. (This figure is kindly given by Prof. Kaori Togashi)

the sperm transport to oviduct, and accept of fertilized embryo for implantation, respectively. ${ }^{3}$ Thus, the peristaltic movement of the junctional zone is essential for reproduction in the human female. Since diffuse leiomyomatosis is characterized by the occurrence of numerous nodules in the junctional zone, it will give serious influences to the reproductive function in women.

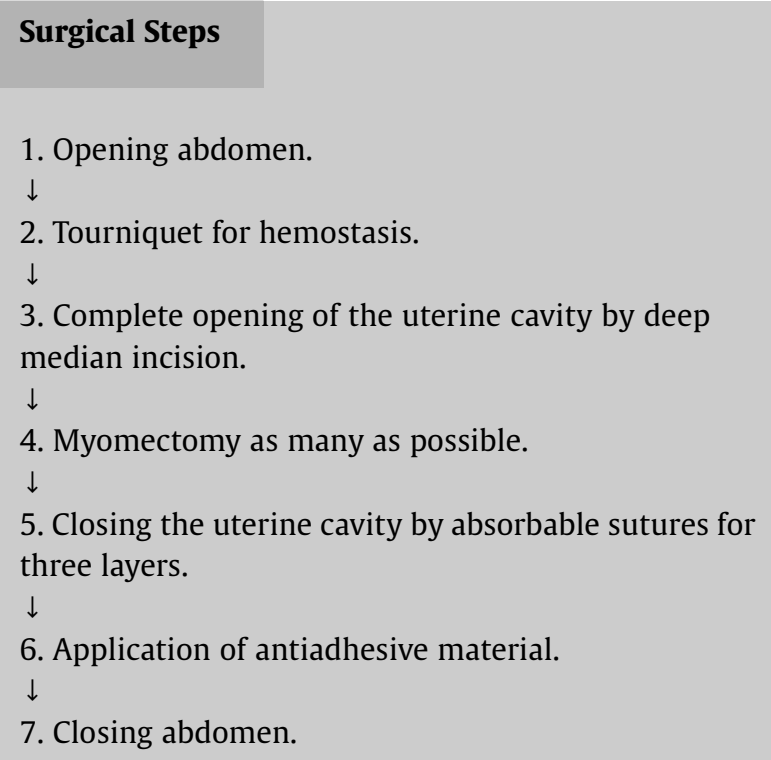

\section{Explanation of Procedures}

General and classical principle for myomectomy described by Dr. Victor Bonney is that only a minimal number of uterine incisions to remove all myomata should be made. This was recommended for performing the complete closure of myometrium. According to the above rule; however, the incision tends to be as small as possible. This should not be the case in the surgery for diffuse leiomyomatosis, since smaller incision will miss the occult presence of minute nodules. Thus, the myometrial incision for diffuse leiomyomatosis should be large enough for complete myomectomy. Here, a novel technique for extensive myomectomy is presented.

\section{(1) Opening Abdomen and Exploration}

Patient is laid in the supine position and urethral catheter is inserted for continuous bladder drainage. The operator incises the abdominal wall longitudinally from the pubis toward the umbilicus, then the fascia and peritoneum. The intestines are softly put upward and maintained with large gauze/sponge, and an appropriate operative field is obtained by the self-retaining retractor. The operative field is kept moist with the Ringer solution, and the tissue is handled gently to avoid unnecessary trauma.

\section{(2) Tourniquet for Hemostasis}

In order to reduce the blood loss during myomectomy, the soft catheter is applied as tourniquet for uterine artery at the level of uterine isthmus (-Fig. 3). To do so, the operator should identify the transparent area of anterior and posterior leaves of broad ligament, and insert the Pean clamps making the holes for tourniquet. The tourniquet will be tied tightly enough to occlude the uterine artery.

\section{(3) Opening the Uterine Cavity}

Deep, median, and longitudinal incision of the uterine corpus

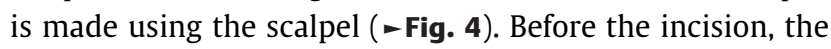
operator should identify the midline that is the midportion between the isthmic ends of each side of Fallopian tube. The incision is made from the fundus to downward till the level of isthmus, first anterior wall and then posterior wall of the uterus. Many myomatous nodules protrude from the cavity and myometrial wall (-Fig. 5). Usually, this procedure is accompanied by small amount of bleeding from the myometrial wall.

\section{(4) Complete Myomectomy}

Almost all of myomatous nodules should be removed from the surface of incised myometrial wall or from the endometrial cavity (-Fig. 6). More than 95\% of macroscopicallydetectable nodules can be removed through this procedure. Blood loss during myomectomy is usually small. It is not necessary for the operator to concern about the preservation of endometrium. Although considerable amount of endometrium is removed during the procedure, it will regenerate and soon recover the normal menstrual cycle. So far, there have been no patients with amenorrhea or Asherman's syndrome after the surgery. 


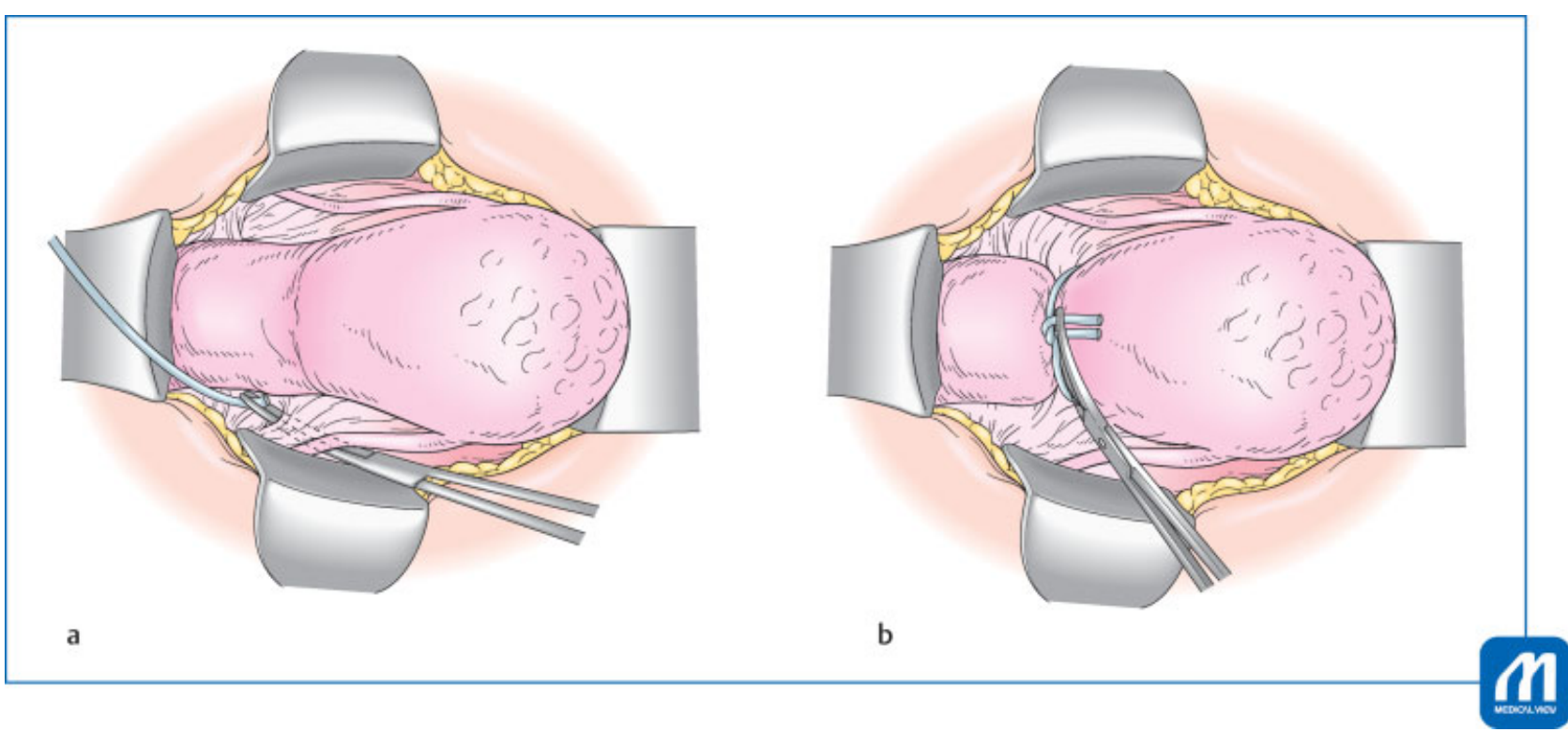

Fig. 3 Tourniquet for Hemostasis: in order to reduce the blood loss during myomectomy, the soft catheter is applied as tourniquet for uterine artery at the level of uterine isthmus. To do so, the operator should identify the transparent area of anterior and posterior leaves of broad ligament, and insert the Pean clamps making the holes for tourniquet(A). Then the soft catheter will be tied(B). (Reproduced with permission from Suzuki and Konishi. ${ }^{1}$ Copyright $\odot$ Medical View).

\section{(5) Closing the Uterine Cavity}

When no remaining tumors are macroscopically visible in the uterine wall, it is time to close the cavity and reconstruct the uterine corpus. Before starting the closing sutures, the tourniquet is once released to check the presence of heavy bleeding from the myometrium. Usually, there is no massive bleeding. Reconstruction of the uterine corpus is performed by the same method as done in the Jones operation for unification of the bicornuate uterus, using three layers of interrupted sutures with 3-0 or 2-0 Vicryl. The sutures are placed at approximately $5-\mathrm{mm}$ intervals. The most inferior

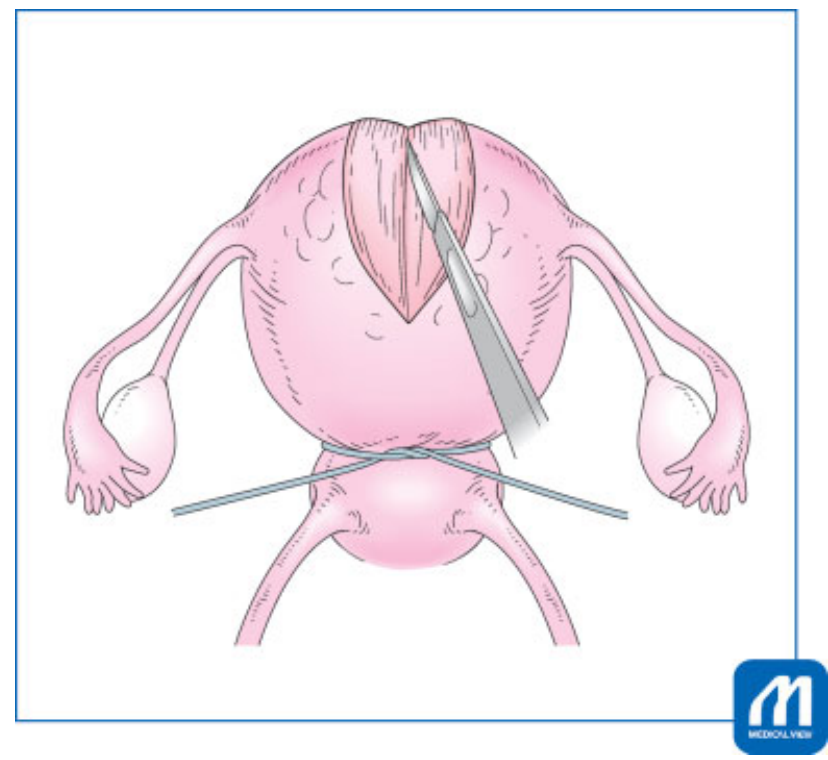

Fig. 4 Opening the uterine cavity: deep, median, and longitudinal incision of the uterine corpus is made using the scalpel. (Reproduced with permission from Suzuki and Konishi. ${ }^{1}$ Copyright $\odot$ Medical View). sutures close to the isthmus should be placed first, and the remainder of the sutures is then placed, gradually approximating the both sides of wall ( - Fig. 7). Importantly, the first layer for subendometrial sutures should not appear on the endometrial cavity. In the second layer, the intramural sutures should be tied not to remain dead spaces. In the third layer for the uterine serosa, the sutures should be placed beautifully.

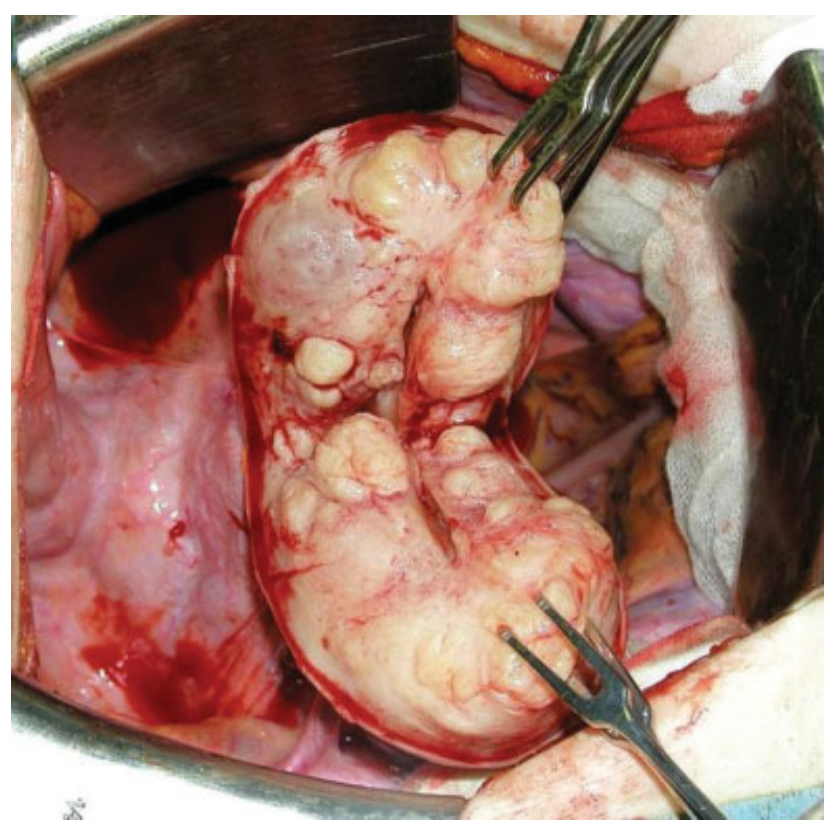

Fig. 5 Opening-cavity findings of diffuse leiomyomatosis in the same patient as shown in - Fig. 1. Numerous nodules protrude from the cavity and myometrial wall. 


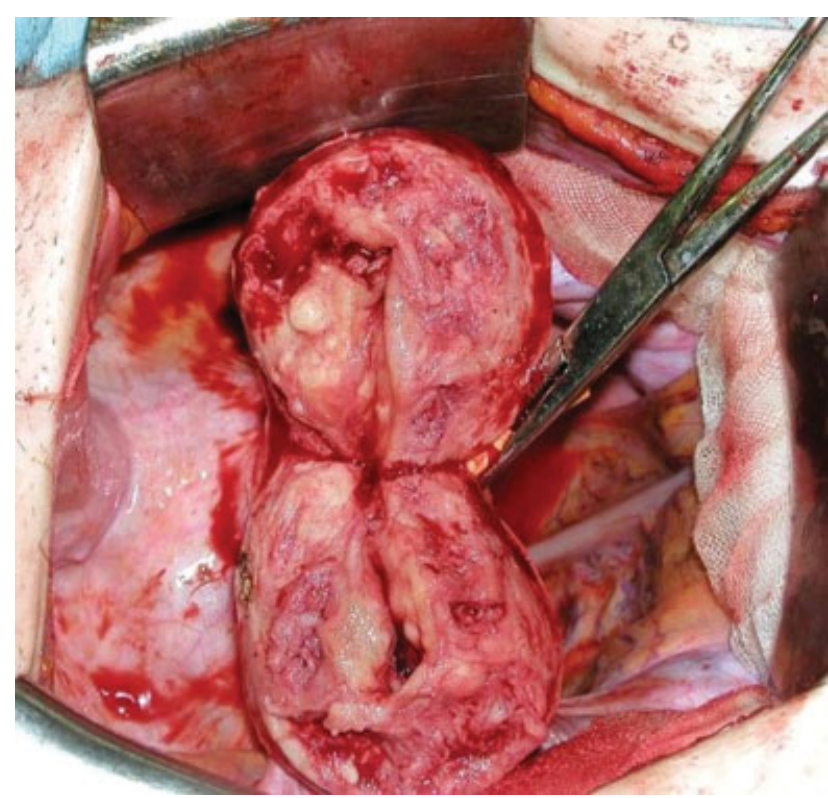

Fig. 6 Complete myomectomy for diffuse leiomyomatosis in the same patient in - Fig. 5. Almost all of nodules are removed from the myometrial wall and from the endometrial cavity.

\section{(6) Application of Antiadhesive Material}

After completing the uterine sutures, the operator should check the bleeding from the uterus, release the tourniquet, and repair the peritoneal holes. Then, the surface of the uterus is covered by antiadhesive materials, such as Seprafilm, Interceed, or Adspray.

\section{(7) Closing Abdomen}

The abdomen is closed with each sutures for the peritoneum, fascia, and skin. The operative technique employed should be consistent with the goal of sparing fertility and the possibility of successful pregnancy.

\section{Postoperative Care and Pregnancy}

A period of 3 months of contraception is sufficient before conception. Usually, patients are prescribed with three cycles of estrogen followed by estrogen-progestin for enhancement of endometrial regeneration. Active fertile treatment, such as in vitro fertilization-embryo transfer (IVF-ET) may be needed for conception. If pregnant, the course should be carefully checked by perinatologist. So far, there have been no experiences of uterine rupture during pregnancy, but Caesarean section is recommended for safe delivery.

\section{Tips and Warnings-Histopathology of Diffuse Leiomyomatosis}

It is very interesting to see the microscopic features of diffuse leiomyomatosis because we can see the scene of early development of leiomyoma. Actually, there are many microscopic nodules of hypercellular tumor cells in the subendometrial zone of normal-appearing myometrium (-Fig. 8). They blended with each other and merged imperceptibly with the surrounding normal myometrial smooth muscle cells. Generally, ordinary leiomyomas are composed of mature smooth muscle cells and immunohistochemically positive for both smooth muscle actin and caldesmon. In contrast, many of smooth muscle cells in diffuse leiomyomatosis show hypercellular and immature features, since they have scant cytoplasm positive for smooth muscle actin but negative for caldesmon but some other cells are positive for both proteins. Thus, in the minute nodules, we can see the process of differentiation of smooth muscle cells in the growth of leiomyomas. ${ }^{4}$

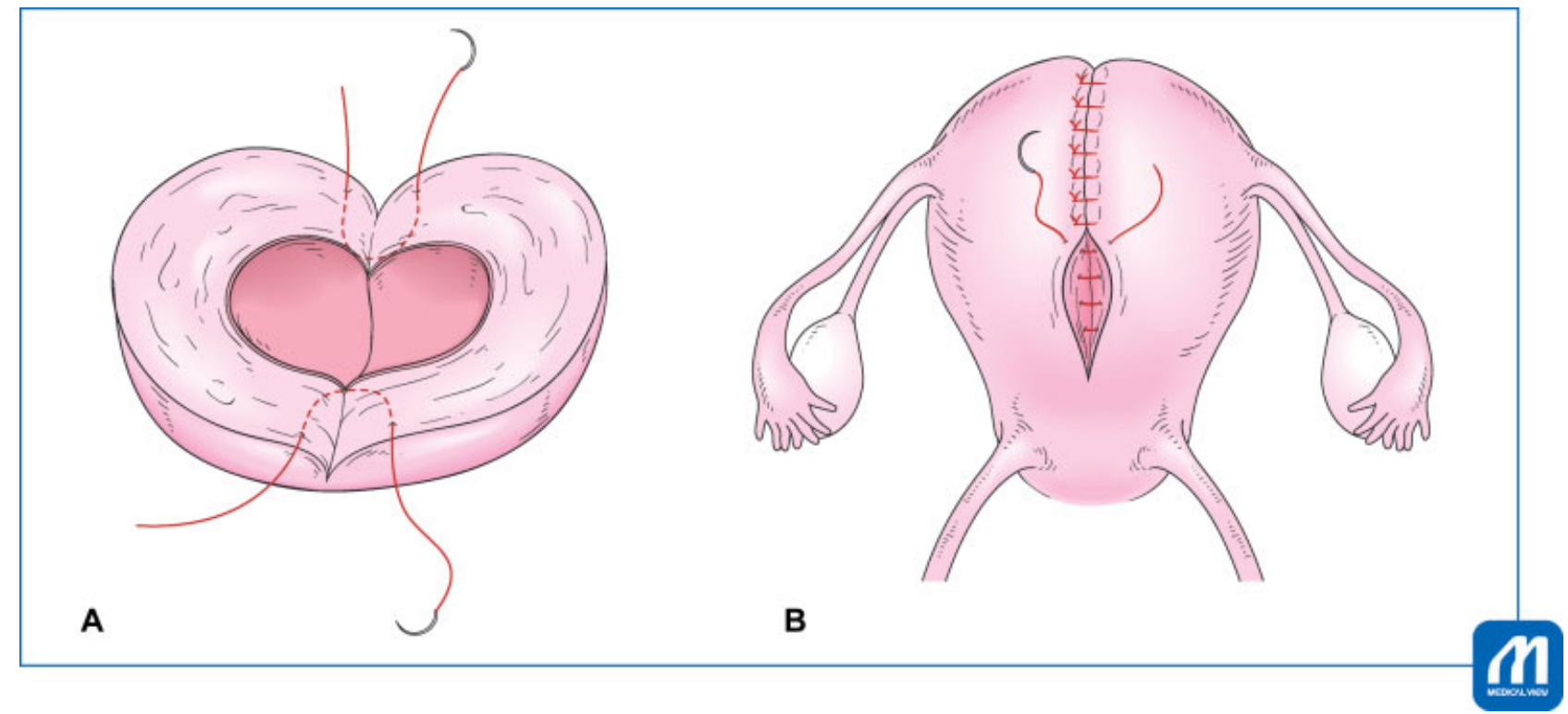

Fig. 7 Sutures of the uterine corpus for reconstruction: the first layer sutures should not appear on the endometrial cavity(A). The third layer sutures for the serosal surface should be placed beautifully(B). (Reproduced with permission from Suzuki and Konishi. ${ }^{1}$ Copyright @ Medical View). 

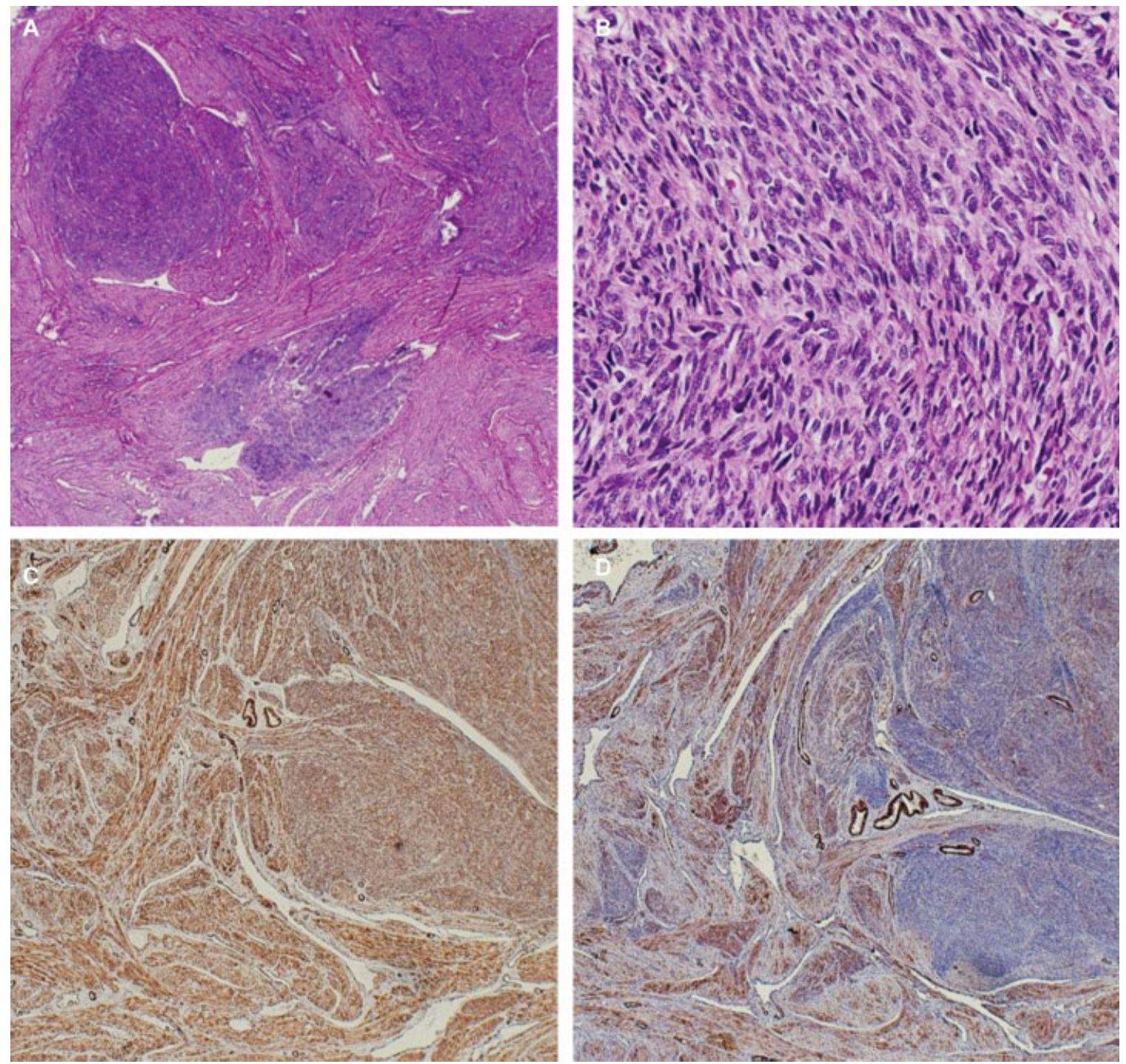

Fig. 8 Microscopic features of diffuse leiomyomatosis: hypercellular smooth muscle cells are irregularly arranged intervening with the surrounding normal smooth muscle cells. HE section of low magnification(A) and higher magnification(B) showing immature smooth muscle cells, which are immunohistochemically positive for smooth muscle action(C), but negative for caldesmon(D). HE, hematoxylin-eosin.
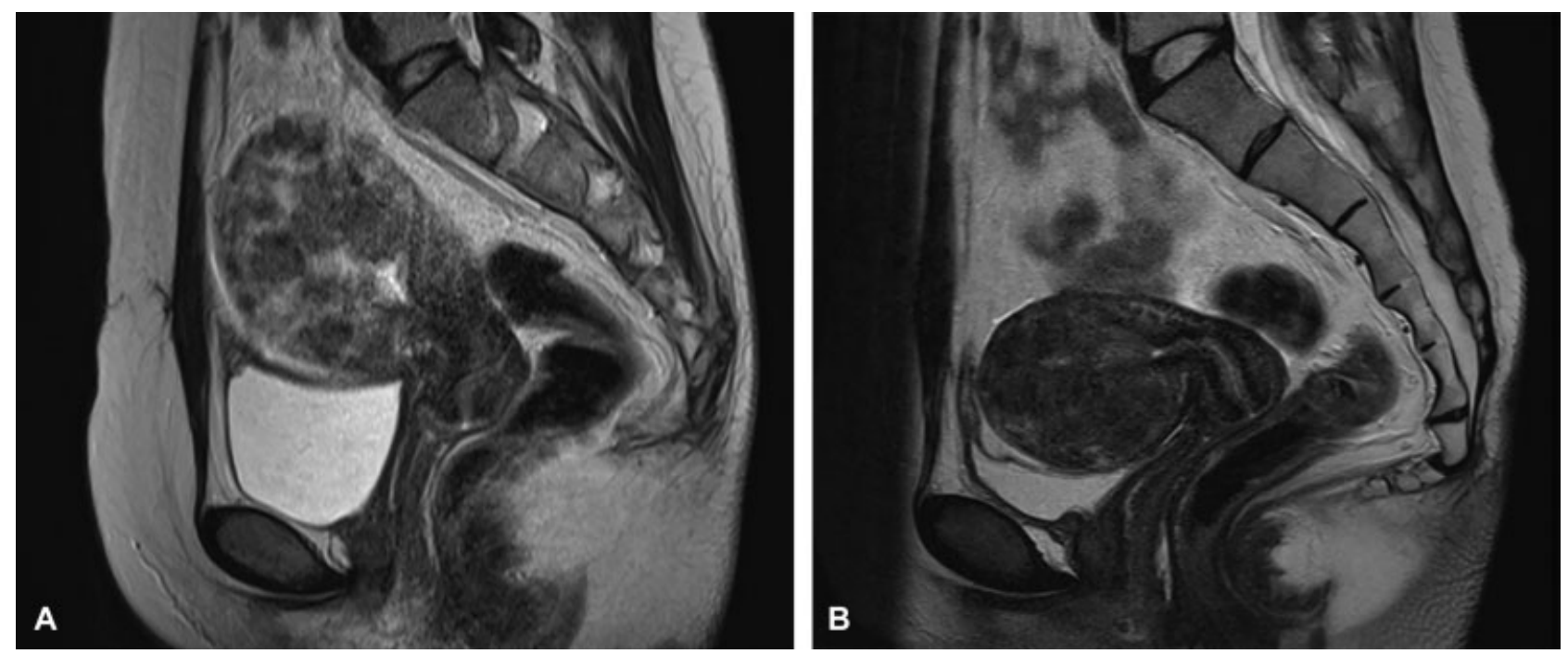

Fig. 9 Case presentation: a 27-year-old woman presented with recurrent hypermenorrhea and past histories of two surgeries, such as open myomectomy and hysteroscopic myomectomy. MRI T2-weighted findings of diffuse leiomyomatosis at presentation(A) and after treatment with four cycles of $\mathrm{GnRH}$ agonist treatment(B). MRI, magnetic resonance imaging. 
Case Presentation: A 27-year-old, nulligravid woman presented with hypermenorrhea and dysmenorrhea. Her past history was GnRH agonist treatment for leiomyoma at 25 years, laparotomic myomectomy at 26 years and then hysteroscopic myomectomy at 27 years of age. She was recently married and desired childbearing. MRI showed numerous small myomatous nodules in the junctional zone ( - Fig. 9). First, she received four cycles of GnRH agonist
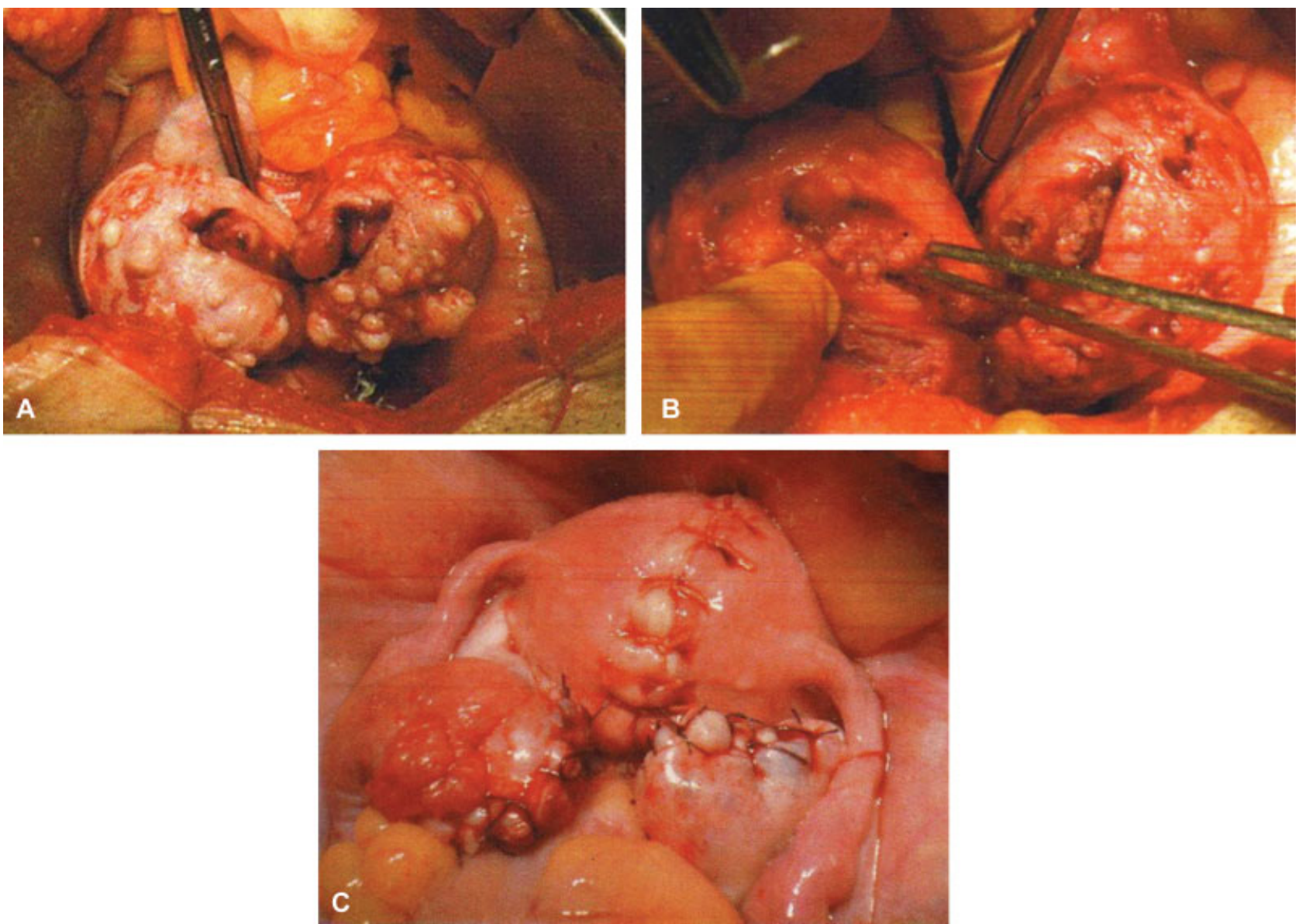

Fig. 10 Case presentation: myomectomy findings of diffuse leiomyomatosis in the same patient, showing the cut section of the uterine corpus (A), the uterine wall after myomectomy (B), and reconstruction of the uterine corpus (C). Six months after the surgery, the patient became pregnant.
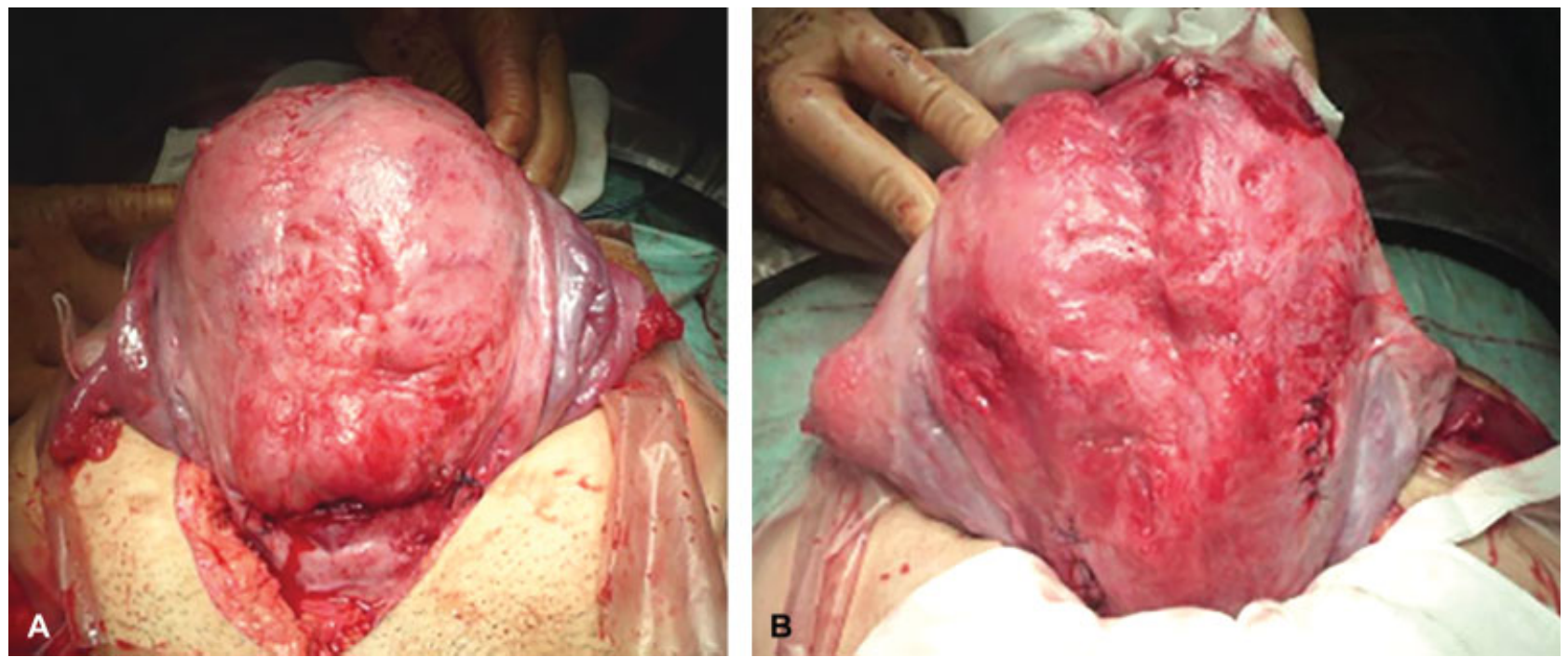

Fig. 11 Caesarean section findings of the same patient in - Figs. $\mathbf{9}$ and 10, showing the anterior view $(\mathbf{A})$ and the posterior view(B) of the uterine corpus just after C-section. Scar of myomectomy is visible, but there are no ruptures. 
treatment, and MRI showed much decrease in size of uterus and modules. She underwent laparotomy and removal of 90 nodules using the current myomectomy method (-Fig. 10). Operating time was 3 hours and the blood loss was $200 \mathrm{~g}$. Six months after the myomectomy, fertile therapy resulted in pregnancy. The course of pregnancy was uneventful, and a male baby $2,174 \mathrm{~g}$ was born by Caesarean section at 36 weeks. There were no ruptures of the uterine wall, and the myomectomy scar was smooth (-Fig. 11).

Conflict of Interest

None.

\section{References}

1 Suzuki A, Konishi I. Myomectomy for diffuse leiomayomatosis. In: Hiramatsu Y, Konishi I, Sakuragi N, Takeda S, eds. Mastering the Essential Surgical Procedures OGS Now, No.11. Uterine Myoma: How to Operate in These Cases? (Japanese). Tokyo: Medical View; 2012:26-33

2 Togashi K, Nakai A, Sugimura K. Anatomy and physiology of the female pelvis: MR imaging revisited.J Magn Reson Imaging 2001; 13(06):842-849

3 Kido A, Togashi K. Uterine anatomy and function on cine magnetic resonance imaging. Reprod Med Biol 2016;15(04):191-199

4 Konishi I, Fujii S, Ban C, Okuda Y, Okamura H, Tojo S. Ultrastructural study of minute uterine leiomyomas. Int J Gynecol Pathol 1983;2(02):113-120 